\title{
Esophageal deformation of Gnathostoma spinigerum in ivermectin-treated rats, and anthelminthic efficacy
}

\author{
M. T. ANANTAPHRUTI ${ }^{1 *}$, M. KOGA ${ }^{2}$, S. NUAMTANONG ${ }^{1}$, R. NINTASEN ${ }^{3}$
}

\begin{abstract}
${ }^{1 *}$ Department of Helminthology, Faculty of Tropical Medicine, Mahidol University, 420/6 Ratchawithi Road, Bangkok 10400, Thailand, E-mail:tmmtr@mahidol.ac.th; ${ }^{2}$ Department of Parasitology, Faculty of Medicine, Kyushu University, Fukuoka, Japan; ${ }^{3}$ Department of Tropical Pathology, Faculty of Tropical Medicine, Mahidol University, Thailand
\end{abstract}

\begin{abstract}
Summary
Ivermectin $2 \mathrm{mg} / \mathrm{kg}$ single dose reduced worm load by $83.3 \%(75.0-91.7 \%)$ for rats experimentally infected with 20 Gnathostoma spinigerum advanced third-stage larvae administered at week 1, with sacrifice at week 15 post-infection. Some larvae recovered from the muscles of treated rats were found to be grossly deformed in appearance. Morphological changes in the Gnathostoma larvae were clearly observed. The internal structures of the deformed larvae showed significant degeneration in the esophageal glandular tissues. The esophageal lumen branched irregularly and occupied a wider area in the center of the esophagus. The epithelial cells thickened along these branches. The esophageal gland ducts were completely destroyed. The excretory ability of the esophageal cell glands may be depressed, causing loss of function. It is suggested that ivermectin is effective against Gnathostoma larvae due to worm recovery reductions. The drug efficacy appears to generate by esophageal destruction of the worms.
\end{abstract}

Key words: Esophagus tissues; Gnathostoma spinigerum; ivermectin; rats; treatment efficacy

\section{Introduction}

Ivermectin has been used from its inception as a veterinary anthelmintic for nematode infections (Campbell, 1985; Fox, 2006). It is an essential drug for the treatment of human strongyloidiasis (Naquira et al., 1989; Suputtamongkol et al., 2008). It is a significant drug in control programs for human filarial diseases (Ottesen et al., 1997; Ciment, 1999; Richard-Lenoble et al., 2003).

Gnathostomiasis, a larval nematode infection, is mainly caused by Gnathostoma spinigerum. Generally, it causes intermittent cutaneous migratory swelling or creeping eruption (Daengsvang, 1980). The larvae sometimes invade the bulbous oculi (Bhattacharjee et al., 2007) and central nervous system, the latter causing potentially fatal infections in humans (Chitanondh \& Rosen, 1967). Ivermectin has been trialed for treating gnathostomiasis in experimental animals. In rabbits infected with $100 \mathrm{G}$. spinigerum advanced third-stage larvae (GsAL3), median worm reduction was $74.2 \%$ (range 58.3 - 86.7\%) when the animals were subcutaneously administered a single dose of ivermectin $0.2 \mathrm{mg} / \mathrm{kg}$, at week 4 and sacrificed at week 28 post-infection (Anantaphruti et al., 1992). White rats subcutaneously injected with ivermectin $2 \mathrm{mg} / \mathrm{kg}$ single dose showed $87.6 \%$ worm reduction; the animals were infected with 30 GsAL3, treated at one week post-infection, and sacrificed at week 20 post-treatment. Abnormal, deformed and shortened worms were found in the ivermectin-treated rats after week 5 of treatment. The number of deformed worms found increased according to the duration after drug administration, up to 15 weeks. At week 20, no live deformed worms were found (Waikagul et al., 1991). The present study compared the histology of the alimentary tracts of deformed $G$. spinigerum larvae found from experimentally infected rats, sacrificed at 15 weeks post-administration of $2 \mathrm{mg} / \mathrm{kg}$ single dose of ivermectin, with larvae obtained from untreated rats. Post-ivermectin treatment worm burdens were also analyzed.

\section{Materials and Methods}

Infective advanced third-stage larvae of Gnathostoma spinigerum (GsAL3) were obtained from the livers of naturally infected eels purchased from local markets in Bangkok, using $1 \%$ acid-pepsin digestion. A total of 6 white rats of both sexes, weighing $135-162 \mathrm{~g}$, were individually infected orally with 20 GsAL3 via polyethylene tube. One week post-infection, 4 rats were administered subcutaneously a single dose of $2 \mathrm{mg} / \mathrm{kg}$ ivermectin (Ivomec injection, $1 \% \mathrm{wv}, \mathrm{MSD}$ ) diluted in polyethylene glycol (PEG), Ivomec : PEG = $1: 1.4$. One more rat served as 
a PEG vehicle control, by subcutaneous injection with 2.8 $\mathrm{ml} / \mathrm{kg}$ of PEG at one week post-infection. Another rat served as an untreated control. All animals were sacrificed by overdose of diethyl ether at 15 weeks' post-infection. The skins were removed and the subcutaneous tissues examined for larvae. The pleural and abdominal cavities were opened, and all viscera removed. The body muscles were separated into four masses; front limbs, hind limbs, chest and abdomen, and back. Each block of muscles was dissected and searched for GsAL3 by pressing between 2 glass plates under a stereo-microscope. All larvae parasitized were gently removed and washed in physiological saline solution. The larvae found in each rat were counted according to the 5 divisions (i.e., subcutaneous tissues and muscles of each 4 block). Worms recovered were analyzed as percent reduction, which was calculated by $100-$ [(worms found in treated rats / worms found in untreated rats) $x$ 100]. Larval appearance and viability were examined under a dissecting microscope.

Recovered deformed larvae were preserved in $10 \%$ neutral buffered formalin, dehydrated by processing in ascending ethanol series, embedded in paraffin, cut into serial crosssections (4 microns in thickness) and the sections were stained with hematoxylin \& eosin. Normal larvae from an untreated, control rat were co-processed with the deformed larvae. Morphological changes, e.g., deformation of the alimentary tract structures of the transformed larvae, were examined microscopically and compared with larvae from the control. The study was approved by the Animal Ethical
Clearance Committee of the Faculty of Tropical Medicine, Mahidol University, Bangkok, Thailand.

\section{Results}

\section{Ivermectin treatment efficacy}

The results for the worms recovered from rats infected with 20 advanced third-stage larvae of Gnathostoma spinigerum (GsAL3) are shown in Table 1. In the control rats, 13 worm larvae were recovered from the untreated rats, and 11 from the PEG control; the average was 12. All larvae found were encysted in the muscles, and appeared normal and viable. For the 4 ivermectin-treated rats, the mean number of larvae found was 2 (range $1-3$ ); larvae were found in the muscles (1.5) and subcutaneous tissues (the inner surfaces of the skin, 0.5) (Table 1A). All larvae recovered were encapsulated, apart from one, which was unencysted (larva) in the rib muscle of treated rat No.4 (Table 1B). The free larva was found deformed in appearance, and short, about $1 / 3$ of normal worm length (Fig.1). An area of the mid-section of the intestine appeared to fold up anteriorly to about $1 / 2$ of the esophagus. One pale encysted larva was recovered from the back muscle of rat No. 1. Other larvae found were viable and normal in appearance. Comparing the control and ivermectin-treated rats, the ratio of larvae found in the muscle: subcutaneous tissue was $12: 0$ in the control, and $3: 1$ in the treated group (Table 1A). The worm-reduction rate for ivermectin in this study was $83.3 \%$ (range $75.0-91.7 \%$ ) (Table $1 \mathrm{~A})$.

Table 1. Number of Gnathostoma spinigerum advanced third-stage larvae (GsAL3) recovered from 6 rats infected with 20 GsAL3, sacrificed at week 15 post-infection; untreated control, vehicle control (PEG) and those treated with ivermectin $2 \mathrm{mg} / \mathrm{kg}$ subcutaneously at one week postinfection.

(A) in subcutaneous tissues and muscles of rats

\begin{tabular}{|c|c|c|c|c|c|c|c|c|}
\hline \multirow{3}{*}{ Sites / organs } & \multicolumn{7}{|c|}{ Number of larvae detected } & \\
\hline & \multicolumn{3}{|c|}{ Control rats } & \multicolumn{5}{|c|}{ Experiment rats } \\
\hline & Untreated & PEG & Average & No. 1 & No. 2 & No. 3 & No. 4 & Average \\
\hline Subcutaneous tissues & 0 & 0 & 0 & 2 & 0 & 0 & 0 & 0.5 \\
\hline Muscles & 13 & 11 & 12 & 1 & 2 & 1 & 2 & 1.5 \\
\hline Total & 13 & 11 & 12 & 3 & 2 & 1 & 2 & 2 \\
\hline Worm reduction rate ${ }^{\mathrm{a}}$ & - & - & - & 75 & 83.3 & 91.7 & 83.3 & 83.3 \\
\hline
\end{tabular}

${ }^{\mathrm{a}}$ Worm reduction rate $=100-[$ (worms found in experiment $/$ worms found in control $\left.) \times 100\right]$

(B) in different sites of muscles of rats

\begin{tabular}{|c|c|c|c|c|c|c|c|c|}
\hline \multirow{3}{*}{ Sites / organs } & \multicolumn{7}{|c|}{ Number of larvae detected } & \\
\hline & \multicolumn{3}{|c|}{ Control rats } & \multicolumn{5}{|c|}{ Experiment rats } \\
\hline & Untreated & PEG & Average & No. 1 & No. 2 & No. 3 & No. 4 & Average \\
\hline Fore limbs & 3 & 4 & 3.5 & 0 & 0 & 0 & 1 & 0.25 \\
\hline Hind limbs & 3 & 1 & 2 & 0 & 1 & 1 & 0 & 0.5 \\
\hline Chest \& abdomen & 0 & 2 & 1 & 0 & 0 & 0 & $1^{\mathrm{b}}$ & 0.25 \\
\hline Back body & 7 & 4 & 5.5 & $1^{\mathrm{c}}$ & 1 & 0 & 0 & 0.5 \\
\hline Total & 13 & 11 & 12 & 1 & 2 & 1 & 2 & 1.5 \\
\hline
\end{tabular}




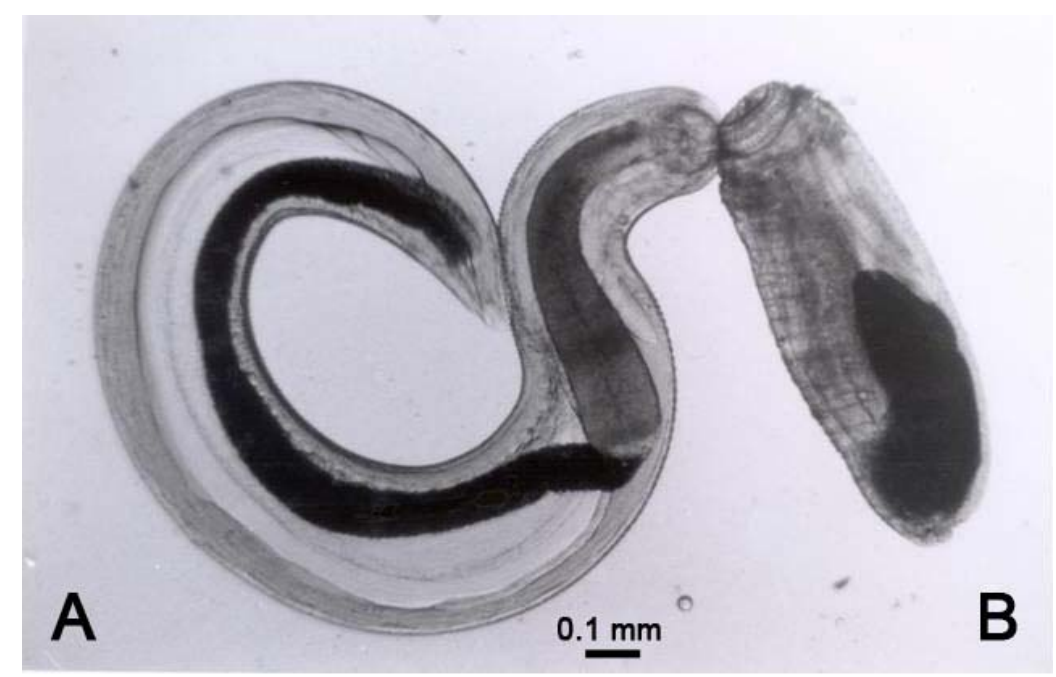

Fig. 1. Gnathostoma spinigerum advanced third-stage larva from untreated control (A) and ivermectin-treated (B) rat. The larva (B) was deformed in appearance; short, about $1 / 3$ of normal worm length (A). The approximately mid-intestine folds up anteriorly to about $1 / 2$ of the esophagus.

\section{Study of esophageal structures}

Two larvae from an untreated control and two deformed larvae from muscle of treated rats (No. 1 and 4) were processed histologically for serial sectioning. A morphological cross-section of the esophageal glandular region of GsAL3 is shown in Fig. 2.

\section{Larvae from control rats}

A normal Gnathostoma larva in H\&E-stained sections usually has small 3-lobed lumens. A small parenchyma occupied the center part of the esophageal coelomic cavity and a thin esophageal epithelium limited the center of the esophageal glandular tissues. The epithelial layer was so thin that it was difficult to detect in normally sectioned specimens. Very rarely, the nuclei could be detected in the

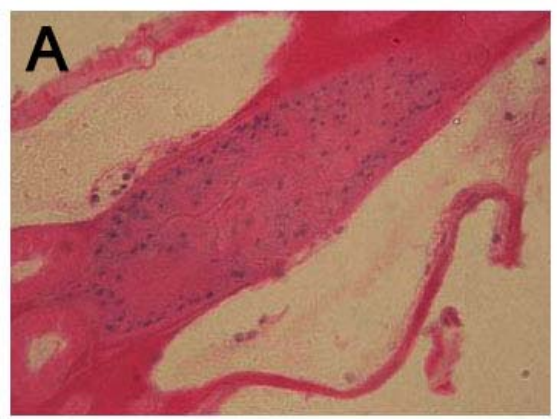

B

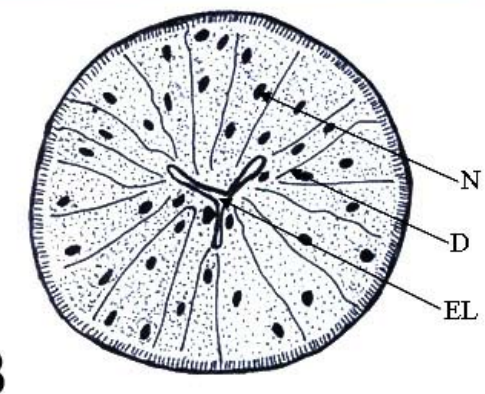

epithelial cells, as described by Morita (1955a). All esophageal glandular ducts and excreting cells, which excrete mucin (Morita, 1955b) were arranged radially, oriented in order, toward the esophageal lumen, and were syncytial in appearance. Many nuclei of the esophageal glandular cells were clearly detected, distributed evenly in each cell, appearing as dense basophilic granules of the esophageal tissues (Fig. 2, A and B).

\section{Larvae from treated rats}

The esophageal structures of 2 grossly deformed Gnathostoma larvae were studied. H\&E-stained section microscopy of the deformed larvae revealed abnormal changes to the esophagus. The esophagus' coelomic cavity was enlarged and occupied a wider area of the central esopha-
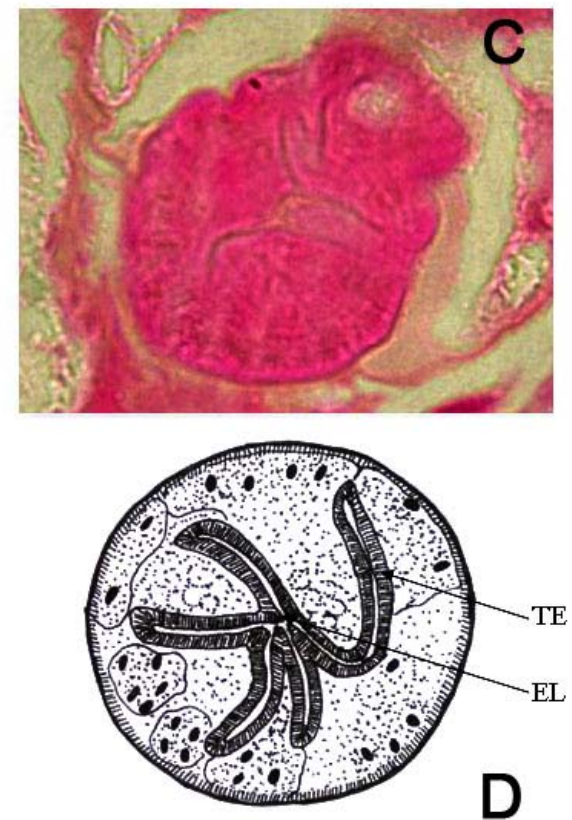

Fig. 2. Microscopic cross-sectioned structures of the esophagus of Gnathostoma spinigerum advanced third-stage larvae from untreated and ivermectin-treated rats, H\&E stain (A and C) and illustration (B and D). N, nucleus of esophageal glandular cell; D, esophageal glandular duct; EL, esophageal lumen; TE, thick epithelium showing thick hyalin degeneration of epithelium of esophageal lumen in treated rat. 
gus. The esophageal lumen branched irregularly into 4 or 5 lobes with some branches reaching the outer membrane of the esophagus. Significant hyaline degeneration was observed in the esophageal glandular tissues. The severely hyaline degenerated esophageal epithelial tissue was thickened markedly along the lumen branches. The cells of the epithelial tissues were arranged irregularly, apparently degenerated and dysfunctional, since the epithelium cell walls had become wider and appeared much darker. The esophageal gland ducts were completely destroyed and residues were rarely seen, which suggested disconnection of the drainage. The glandular cell membranes became round, rectangular, or irregular in shape. Most of the glandular cells had hyaline and swollen degenerations and their normal ability had obviously collapsed where many ducts were faded. Gland nuclei were still evident, but they remained only in the outer edges of the esophagus (Fig. 2, C and D). In some sections, the gland nuclei had mostly disappeared, or some glandular cells had no nucleus, which meant that all areas of the cells had undergone dysfunction. The secretory ability of the esophageal glands seemed to have been completely lost. An unencysted, shortened larva from rat No.4 showed wider damaged esophageal areas than the pale encysted larva from rat No. 1. The apparently normal (from the external view), viable larvae were not examined because it seemed difficult for the drug to invade the capsules after they had completely encapsulated.

\section{Study of intestinal structures}

In the intestinal region, cross-sections of worm showed a single layer of simple columnar epithelial cells of the intestinal epithelium, which appeared somewhat thicker than the controls. However, no differences in intestinal-cell structures were apparent between the worms in the two groups, untreated and treated.

\section{Discussion}

The standard antiparasitic dose of ivermectin is $0.2 \mathrm{mg} / \mathrm{kg}$ (Campbell, 1985; Bennett et al., 1988). In human gnathostomiasis, cure rates of 95.2 and $76 \%$ were reported using the standard dose (Nontasut et al., 2000; Kraivichian et al., 2004). Nevertheless, in an experimental study of animal gnathostomiasis, a 10-fold higher dose showed better efficacy. A higher worm reduction rate $(84.2 \%)$ was found in rabbits treated with $2 \mathrm{mg} / \mathrm{kg}$, compared with 74.2 $\%$ with $0.2 \mathrm{mg} / \mathrm{kg}$ (Anantaphruti et al., 1992). A similar worm reduction rate $(87.6 \%)$ was obtained when a dose of $2 \mathrm{mg} / \mathrm{kg}$ was administered to rats infected with gnathostomiasis (Waikagul et al., 1991). The above findings support the use of $2 \mathrm{mg} / \mathrm{kg}$ in the current study, where a wormreduction rate of $83.3 \%$ was found.

Gnathostoma spinigerum advanced third-stage larvae (GsAL3) are usually found encysted in the muscles of laboratory animal hosts (Daengsvang, 1980; Anantaphruti et al., 1986). Other sites may include the subcutaneous tissues of the skin or the visceral organs (Waikagul et al., 1991). Unencysted, free larvae have infrequently been found in these tissues (Daengsvang, 1980). Waikagul et al. (1991) found larvae in the muscles, subcutaneous tissues, and visceral organs, at a ratio of approximately $10: 1$ : 0.14 . In our study, all larvae were found only in the muscles of untreated rats, with none in the subcutaneous tissues or visceral organs. In the muscles, larvae were distributed throughout the body, but with more in the muscles of the back and hind limbs.

In the ivermectin-treated rats, a number of larvae migrated to the subcutaneous tissues. We found the ratio of larvae in muscles to subcutaneous tissues was $3: 1$ in the treated group, and $12: 0$ in the control group, whereas it was 1.9: 1 and $9.8: 1$ in the study by Waikagul et al. (1991). It seems likely that the worm recovery rate for our study was affected by the small number of experimental animals available, and may thus be insufficient to draw statistically definitive conclusions. Nevertheless, our data corresponded well with the study by Waikagul et al., 1991. In addition, the length of time between treatments to sacrifice of the animals might affect the allocation of the larvae. In the study by Waikagul et al. (1991), worm recovery obtained from duration ranged from $5-20$ weeks, while it was 15 weeks in our experiment. In humans, $0.2 \mathrm{mg} / \mathrm{kg}$ single-dose ivermectin treatment has proven effective for gnathostomiasis, though with varying cure rates of 95.2 and $76 \%$, respectively (Nontasut et al., 2000; Kraivichian et al., 2004). Furthermore, ivermectin tended to stimulate the outward migration of larvae, close to the skin surface, where they may be seen and removed (Nontasut et al., 2000). The outward-migration stimulating phenomenon in the above study suggests a relationship with the current study, as the subcutaneous : muscle larval ratio was higher $(1: 3)$ in the ivermectin-treated, vs. $0: 12$ in the control rats, although the small number did not permit calculation of any statistical difference. The reason for this phenomenon is unclear. However, this finding for ivermectin is supported by earlier work on albendazole therapy in humans and mebendazole treatment for animals. After albendazole 400 - $800 \mathrm{mg}$ per day was administered for $14-21$ days to gnathostomiasis patients, the larvae moved closer to the skin surface, observed as a small nodule, red pimple or red line and were extracted from some patients, whereas none was seen in placebo-treated patients (Suntharasamai et al., 1992). A similar finding was investigated in 30 GsAL3-infected rats treated with $10 \mathrm{mg} / \mathrm{kg}$ sequential mebendazole therapy for a total of 21 and 28 doses. Stimulation of migration activity was evident during medication, during which larvae were found penetrating the skin (Waikagul et al., 1997).

Ivermectin stimulates the release of GABA ( $\gamma$-amino butyric acids) from the nerve endings and enhances the binding of GABA to its receptor, which is on the postsynaptic membranes of an excitatory motor-neuron in nematodes. Enhanced GABA binding results in an increased flow of chloride ions into the cells, with consequent hyper-polarization and blocking of signal transmission from the central command interneurons to the peripheral motor neurons. Therefore, ivermectin immobilizes 
and/or paralyzes the worms (Campbell, 1985; Wang \& Pong, 1982). This supported our finding that the shortened gross appearance of the worm might be due to spasmodic paralysis. Ivermectin seemed to act directly on the esophageal glands, leading to cessation of the esophageal excretion function. The worms finally lost viability and subsequently disappeared from the host bodies. The half-life of oral ivermectin is $<12$ hours in humans and intravenous ivermectin is 1 day in rats (MSD, 1988); nevertheless, their effect on the worms was long-lasting. Waikagul et al. (1991) found deformed Gnathostoma larvae initially at week 5 , then up to week 15 post-ivermectin administration. These data thus indicated that histopathological changes in the worms should be studied later, which was supported by our own study at week 15 post-drug administration.

The main symptom of human gnathostomiasis is cutaneous swelling due to larval migration. During their invasion, worms probably secrete substances toxic to the affected tissues. Since the esophagus is the largest excretory gland, Morita (1955b) collected only the esophagi from larvae. He injected the esophageal extracts into the abdominal skin of rats, where they attracted many eosinophils, some of which destroyed cytomembranes, resulting in degeneration of the affected tissues. The larval esophagus extract was composed mostly of mucin. Thus, he concluded that mucin was the main substance produced by the esophageal gland cells of the worm. Consequently, crude water extract and excretory-secretory products of the larvae exhibited more than 20 polypeptides that were immunogenic with human gnathostomiasis sera (Tuntipopipat et al., 1993). Among these, a 24-kDa protein was described as the specific component (Tapchaisri et al., 1991). Moreover, Nopparata et al. (1992) reported that the proteins extracted from the esophagi and intestines of larvae were more immunogenic than those from the head and body cuticle. They also found that the specific $24-\mathrm{kDa}$ component was present in the esophagi and intestines of the larvae. Mucin, as well as the proteins described by these investigators, might induce regional inflammation with severe eosinophilic and lymphocytic infiltrations. Daengsvang (1980) noted that most gnathostomiasis patients exhibited eosinophilia. About 90 $\%$ of cutaneous gnathostomiasis cases had absolute eosinophil counts $>500$ cells/microliter (Kraivichian et al., 2005). Eosinophil levels reduced to normal within 6 months post-treatment with $0.2 \mathrm{mg} / \mathrm{kg}$ single dose of ivermectin in 20 cured gnathostomiasis cases (Nontasut et al., 2000). It seems likely that the decrease in eosinophils was due to reduced esophageal toxin post-ivermectin administration.

It may be concluded that ivermectin is effective against advanced 3rd-stage larvae of G. spinigerum, based on reductions in worm recovery post-administration. The drug's efficacy may derive from its effect on the structure and function of the esophagus. To evaluate the histological efficacy of this drug with gnathostome larvae, we ought to have verified the deformation of the esophagus in both in vivo and in vitro experiments, however, this study was limited to in vivo. Further study is required, to verify the histological deformation of the esophagus in vitro.

\section{References}

Anantaphruti, M. T., Nuamtanong, S., Waikagul, J. (1992): Effect of ivermectin on experimental gnathostomiasis in rabbits. Trop. Med. Parasitol., 43: $65-67$

Anantaphruti, M. T., Waikagul, J., Nithi-Uthai, S., Pubampen, S., RojekitTikHun, W. (1986): Detection of humoral immune response to Gnathostoma spinigerum in mice. Southeast Asian J. Trop. Med. Public Health, 17: $172-176$

Bennett, J. L., Williams, J. F., DAVe, V. (1988): Pharmacology of Ivermectin. Parasitol. Today, 4: $226-228$

BhattacharJeE, H., Das, D., MEDHI, J. (2007): Intravitreal gnathostomiasis and review of literature. Retina, 27: $67-73$

CAmpbell, W. C. (1985): Ivermectin: An update. Parasitol. Today, 1: $10-16$

Chitanondh, H., Rosen, L. (1967): Fatal eosinophilic encephalomyelitis caused by the nematode Gnathostoma spinigerum. Am. J. Trop. Med. Hyg., 16: 638 - 645

CIMENT, J. (1999): WHO celebrates triumph over river blindness. British Med. J., 319: 1090

Daengsvang, S. (1980): A Monograph on the Genus Gnathostoma and Gnathostomiasis in Thailand. Southeast Asian Medical Information Center, Tokyo

FoX, L.M. (2006): Ivermectin: uses and impact 20 years on. Curr. Opin. Infect. Dis., 19: 588 - 593

Kraivichian, K., Nuchprayoon, S., Siriyasatien, P., SAKSIRISAMPHAN, W., NUCHPRAYOON, I. (2005): Resolution of eosinophilia after treatment of cutaneous gnathostomiasis. J. Med. Assoc. Thai., 88 Suppl 4: S163 - S166 Kraivichian, K., Nuchprayoon, S., Sitichalernchai, P., Chaicumpa, W., Yentakam S. (2004): Treatment of cutaneous gnathostomiasis with ivermectin. Am. J. Trop. Med. Hyg., 71: 623-628

MoritA, T. (1955a): A study on the third stage larva of Gnathostoma spinigerum. I. Morphological features. Acta Med., 25: $411-431$

MoritA, T. (1955b): A study on the third stage larvae of Gnathostoma spinigerum. II Effect of emulsion of larval body, particularly the esophagus gland on the skin of the host. Acta Med., 25: 432 - 446

MSD (Merck Sharp \& Dohme) (1988): Poison Control Monograph. ivermectin. Division of Merck \& Co Ltd, West Point, Pennsylvania

Naquira, C., Jimenez, G., Guerra, J. G., Bernal, R., NALIN, D. R., NEU, D. AzIZ, M. (1989): Ivermectin for human strongyloidiasis and other intestinal helminths. Am. J. Trop. Med. Hyg., 40: $304-309$

Nontasut, P., Bussaratid, V., Chullawichit, S., ChaROENSOOK, N. VISETSUK, K. (2000): Comparison of ivermectin and albendazole treatment for gnathostomiasis. Southeast Asian J. Trop. Med. Public Health, 31: 374 - 377 Nopparatana, C., Chaicumpa, W., TAPChaisri, P., Setasuban, P., RuAngkunaporn, Y. (1992): Towards a suitable antigen for diagnosis of Gnathostoma spinigerum 
infection. Inter. J. Parasitol., 22: 1151 - 1156

Ottesen, E. A., Duke, B. O., Karam, M., Behbehani, K. (1997): Strategies and tools for the control/elimination of lymphatic filariasis. B. World Health Organ., 75: 491 - 503

Richard-Lenoble, D., Chandenier, J., GAXotTe, P. (2003): Ivermectin and filariasis. Fund. Clin. Pharm., 17: $199-203$

Suntharasamai, P., Riganti, M., Chittamas, S., DesaKORN, V. (1992): Albendazole stimulates outward migration of Gnathostoma spinigerum to the dermis in man. Southeast Asian J. Trop. Med. Public Health, 23: 716 - 722 SuputTamongkol, Y., KungPanichKul, N., SilPaSAKORN, S., BEECHING, N. J. (2008): Efficacy and safety of a single-dose veterinary preparation of ivermectin versus 7day high-dose albendazole for chronic strongyloidiasis. Int. J. Antimicrob. Ag., 31: $46-49$

TAPChaisri, P., Nopparatana, C., Chaicumpa, W., SetAsubAn, P. (1991): Specific antigen of Gnathostoma spinigerum for immunodiagnosis of human gnathostomiasis. Inter. J. Parasitol., 21: 315 - 319

Tuntipopipat, S., ChaWENGKIRTTIKUl, R., SiRISINHA, S. (1993): A simplified method for the fractionation of Gnathostoma-specific antigens for serodiagnosis of human gnathostomosis. J. Helminthol., 67: 297 - 304

Waikagul, J., Anantaphruti, M. T., Nuamtanong, S., NithiUTHAI, S. (1991): Study on efficacy of ivermectin against Gnathostoma spinigerum (Nematoda: Spiruroidea) advanced third stage larva in rats. Thai J. Vet. Med., 21: 91 $-103$

Waikagul, J., Nuamtanong, S., Anantaphruti, M. T., PubAMPEN, S., VisiadsuK, K., TAWATSin, A. (1997): Sequential mebendazole treatment against Gnathostoma spinigerum in rat. J. Trop. Med. Parasitol., 20: $40-46$ WANG, C. C., Pong, S. S. (1982) Actions of avermectin B1a on GABA nerves. Prog. Clin. Biol. Res., 97: 373 - 395 